tion in size, making them amenable to surgery.

We present a case of a 56-year-old man presented with a 6 year history of severe right-sided sharp lancinating pain affecting the mandibular division of the trigeminal sensory distribution. On examination there appeared to be a trigger zone over his right zygoma. There was no evidence of any neurological deficit. He was maintained on $1200 \mathrm{mg}$ of carbamezapine a day with little effect.

High-resolution magnetic resonance imaging (MRI) and gadolinium enhanced magnetic resonance angiography (MRA) revealed a very large vascular loop of the right superior cerebellar artery (SCA) passing inferiorly and causing distortion of the root entry zone of the right trigeminal nerve.

Cerebral angiography confirmed the presence of a posterosuperior vermian AVM fed by the right SCA and draining. into the vein of Galen [Figure 1]. Endovascular embolization was performed using Berenstein liquid coils. This resulted in significant reduction in blood flow with transit time through the AVM doubled. Follow-up MRI and MRA with gadolinium after 6 months showed partial patency of the AVM with dilatation of tentorial veins bilaterally and a large loop of SCA on the right. Repeat angiography and embolization showed no change in the appearance of the superior vermian AVM with its three feeders (two from the SCA and one from PICA). Using the same technique catheter was inserted into the SCA and the superior of the two feeders. A straight Berenstein liquid coil was injected. This produced gratifying reflux up the pedicle on check injection and the flow was clearly reduced further [Figure 2]. Despite many attempts it was not possible to catheterize the second feeder. The procedure was terminated without any neurological complication. The patient was discharged after 3 days.After a follow up of 18 months the patient remain free from pain and there was a significant re-

\title{
Arteriovenous malformation presenting with trigeminal neuralgia and treated with endovascular coiling
}

Sir,

Areteriovenous malformations (AVMs) are known to cause trigeminal neuralgia (TGN) ${ }^{[1]}$ The incidence of posterior fossa AVM is less than $10 \%$ of all intracranial AVMs. The association of an AVM and TGN is rare. ${ }^{[2]}$ Eisenbrey and Hegarty were the first to describe a case of AVM presenting with TGN in $1956 .{ }^{[3]}$ Staged endovascular coiling as an adjunct to surgery for large $(3 \mathrm{~cm})$ AVMs can achieve satisfactory reduc-

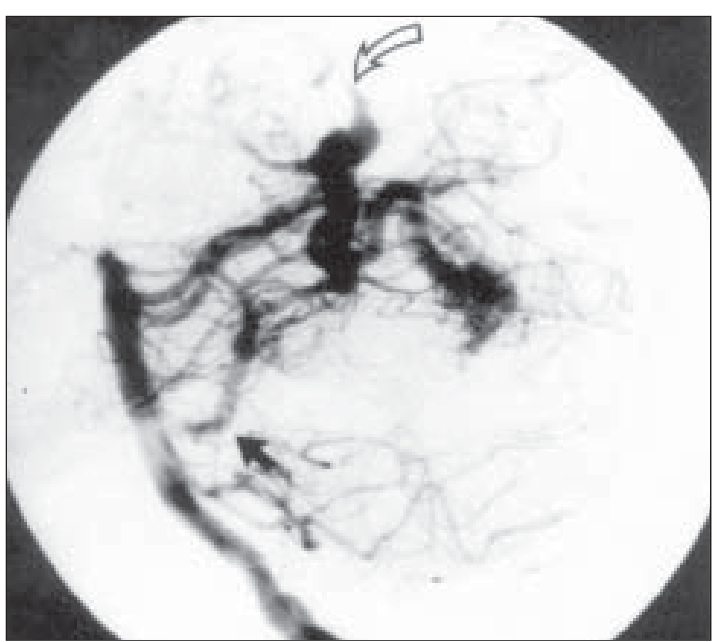

Figure 1: Fight vertebral angiogram showing dilated, ectatic right superior cerebellar artery (solid arrows) and occlusion of the vein of Galen (curved open arrow). 


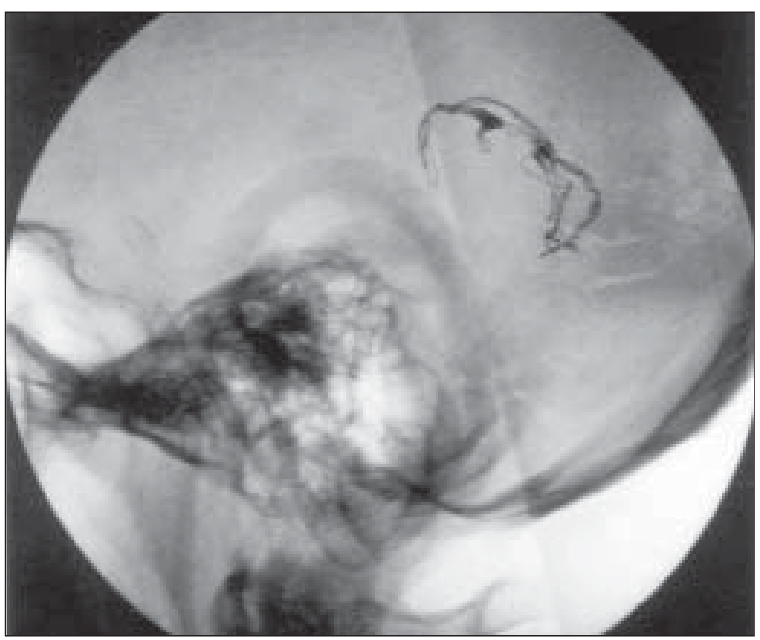

Figure 2: Fight vertebral angiogram following first embolization showing marked reduction in A-V shunting. The deposition of coils is clearly shown.

duction in the volume of the nidus. This case indicates the feasibility of embolization for TGN in selected patients. In this case the angioarchitecture meant that liquid embolic agents such as cyanoacrylate glue were contraindicated. The aim of the procedure was to reduce flow in the AVM with coil embolization. Large AVMs can be treated with staged embolization and surgery (operative surgery or radiosurgery) if needed. A multimodality approach (embolization, radiosurgery, surgical exploration) is the current policy for treating AVMs and takes into account the cumulative risk of bleeding, the location and size of the AVM and the presence of venous

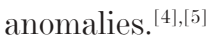

\section{T. C. Athanasiou, S. Nair, H. B. Coakham,}

T. T. Lewis ${ }^{1}$

Department of Neurosurgery and ${ }^{1}$ Neuroradiology, Frenchay Hospital, Bristol, UK. E-mail: tathan5253@aol.com

\section{References}

1. Edwards RJ, Clarke Y, Renowden SA, Coakham HB. Trigeminal neuralgia caused by microarteriovenous malformations of trigeminal nerve root entry zone: Symptomatic relief following complete excision of the lesion with nerve root preservation. J Neurosurg 2002,97:874-80.

2. Kikuchi K, Kamisato N, Sasanuma J, Watanabe K, Kowada M. [Trigeminal neuralgia associated with posterior fossa arteriovenous malformation and aneurysm fed by the same artery. Case report]: Neurol Med Chir (Tokyo) 1990;30:918-21.

3. Eisenbrey AB, Hegarty WM. Trigeminal neuralgia and arteriovenous aneurysm of the cerebellopontine angle. J Neurosurg 1957;13:647-9.

4. Mathis JA, Barr JD, Horton JA, Jungreis CA, Lunsford LD, Kondziolka DS, et al. The efficacy of particulate embolization combined with stereotactic radiosurgery for treatment of large arteriovenous malformations of the brain. A.JNR Am J Neuroradiol 1995;16:299-306.

5. Goto K, Uda K, Ogata N. Embolization of Cerebral Arteriovenous Malformations (AVMs) - Material Selection, Improved Technique, and Tactics in the initial Therapy of cerebral AVMs. Neurol Med Chir (Tokyo) 1998;38:193-9.

Accepted on 27-12-2004 